\title{
First-line fluorouracil-based chemotherapy for patients with severe peritoneal disseminated gastric cancer
}

\author{
Satoru Iwasa - Takako Eguchi Nakajima - Kenichi Nakamura - Atsuo Takashima • \\ Ken Kato · Tetsuya Hamaguchi • Yasuhide Yamada • Yasuhiro Shimada
}

Received: 18 October 2010/Accepted: 18 April 2011/Published online: 15 May 2011

(c) The International Gastric Cancer Association and The Japanese Gastric Cancer Association 2011

\begin{abstract}
Background Treatment with an oral fluoropyrimidine plus cisplatin is widely used for advanced gastric cancer, but patients with severe peritoneal metastasis often cannot tolerate such treatment, due to inadequate oral intake or massive ascites. The aim of this study was to assess the efficacy and safety of systemic chemotherapy for advanced gastric cancer with severe peritoneal metastasis.

Methods The cases of 92 patients with advanced gastric cancer and severe peritoneal metastasis who received firstline chemotherapy at our hospital between May 2001 and February 2007 were retrospectively analyzed. Severe peritoneal metastasis was defined as massive ascites or inadequate oral intake due to peritoneal dissemination. Inadequate oral intake was defined as having required an intravenous drip infusion.

Results All 92 patients received 5-fluorouracil (5-FU)based chemotherapy; 40 of the patients had massive ascites, 34 had inadequate oral intake, and the remaining 18 had both conditions. Among the 86 patients having assessable ascites, 23 (27\%) patients showed an improvement in ascites. Of the 52 patients with inadequate oral intake, $17(33 \%)$ patients improved to the point of ingesting without intravenous drip infusion after receiving the chemotherapy. Median time to treatment failure and
\end{abstract}

S. Iwasa - T. E. Nakajima $(\bowtie) \cdot$ K. Kato - T. Hamaguchi ·

Y. Yamada $\cdot$ Y. Shimada

Gastrointestinal Oncology Division, National Cancer Center

Hospital, 5-1-1 Tsukiji, Chuo-ku, Tokyo 104-0045, Japan

e-mail: tnakajima@marianna-u.ac.jp

K. Nakamura · A. Takashima

Clinical Trials and Practice Support Division, Center for Cancer

Control and Information Services, National Cancer Center

Hospital, Tokyo, Japan overall survival time were 1.9 months [95\% confidence interval (CI) 1.3-2.5 months] and 4.6 months (95\% CI 3.9-5.3 months), respectively. Major grade 3 or 4 adverse events were anorexia (26\%), neutropenia (26\%), and anemia $(22 \%)$.

Conclusion The treatment regimen of 5-FU-based chemotherapy for advanced gastric cancer with severe peritoneal metastasis was feasible, but its efficacy was not sufficient.

Keywords Gastric cancer - Peritoneal metastasis · Ascites · Oral intake

\section{Introduction}

Gastric cancer can spread through various routes, such as by local extension of direct serosal invasion, the lymphatic pathway, and the hematogenous route. Peritoneal metastasis can be disseminated from T3/T4 gastric cancer cells breaking through the serosa. Peritoneal metastasis is reported to be a common reason for the unresectability of gastric cancer [1], and it is also reported to be a common pattern of recurrence after curative resection [2-4]. Peritoneal metastasis causes serious clinical complications such as intestinal obstruction, massive ascites, and hydronephrosis. These complications are associated with abdominal pain, abdominal fullness, and vomiting, resulting in an extremely poor quality of life for the patients.

Recently, an oral fluoropyrimidine plus cisplatin regimen was demonstrated as a standard regimen against advanced gastric cancer in several large clinical Phase III trials [5-7]. However, in these trials, patients with severe peritoneal metastasis, such as those with massive ascites and intestinal obstruction, were excluded from the 
eligibility criteria because they were unable to tolerate hydration, as required during cisplatin-containing chemotherapy, or continue to receive stable oral fluoropyrimidine chemotherapy.

We retrospectively investigated a regimen of chemotherapy, and its safety and efficacy for gastric cancer patients with severe peritoneal metastasis to determine the indications for chemotherapy and to consider the most suitable regimen.

\section{Patients and methods}

Patients

Patients who received first-line chemotherapy for gastric cancer at the National Cancer Center Hospital, Tokyo, between May 2001 and February 2007 were retrospectively recruited for this study according to the following criteria: histologically proven gastric adenocarcinoma; unresectable or recurrent disease; histologically or radiologically confirmed severe peritoneal metastasis; and no prior chemotherapy (adjuvant chemotherapy completed more than 6 months before recruitment was allowed) or radiotherapy. We defined severe peritoneal metastasis as massive ascites or inadequate oral intake due to peritoneal dissemination. We defined inadequate oral intake as requiring and actually receiving an intravenous drip infusion. Patients who were administered an intravenous drip infusion for the purpose of renal protection or as a drug administration route, such as for analgesics, were excluded.

Ascites was classified in four levels: none; mild; moderate; and massive. "None" was defined as undetected by computed tomography (CT) scan; "mild" ascites was localized in only one area such as the pelvic cavity or surface of the liver; "moderate" ascites did not correspond to either mild or massive ascites; and :massive" ascites extended throughout the abdominal cavity.

This retrospective study was approved by the Institutional Review Board of the National Cancer Center and was conducted in accordance with the ethical principles stated in Japan's Ethics Guidelines for Epidemiological Research.

Assessment of response and toxicity

Response in ascites was evaluated as follows: "disappearance" was defined as being undetected by CT scan, "decrease" was defined as decreasing by more than one level, "no change" was defined as remaining at the pretreatment level, and "progression" was defined as deteriorating to a more severe level or becoming clinically worse with more frequently required drainage of ascites. We evaluated the response rate in ascites without interval confirmation. Oral intake improvement was defined as maintaining an adequate amount of intake for 7 days or more without an intravenous drip infusion. Time to treatment failure (TTF) was defined as the interval between the start of treatment and the earliest date among disease progression (either radiologic or symptomatic progression), treatment discontinuation, or death due to any cause. Overall survival (OS) was measured from the start of treatment to the date of death or last follow-up. Survival curves were estimated using the Kaplan-Meier method. All statistical analyses were performed using Dr. SPSS II software (SPSS Japan, Tokyo, Japan). Toxicity was assessed using the National Cancer Institute Common Terminology Criteria for Adverse Events version 3.0.

\section{Results}

\section{Patient characteristics}

A total of 92 patients with severe peritoneal disseminated gastric cancer received systemic chemotherapy between May 2001 and February 2007. The patient characteristics are summarized in Table 1. About half of the patients had a baseline Eastern Cooperative Oncology Group performance status of 2 or greater.

\section{Chemotherapy}

All patients received 5-FU-based chemotherapy regimens. The regimens consisted of methotrexate (MTX)/5-FU in 64 patients, 5-FU continuous infusion (ci) in 21 patients, lowdose 5-FU in 6 patients, and 5-FU/l-leucovorin $(l-\mathrm{LV})$ in 1 patient. The MTX/5-FU regimen consisted of a weekly MTX dose $\left[100 \mathrm{mg} / \mathrm{m}^{2}\right.$, intravenously (iv)] followed by 5-FU $\left(600 \mathrm{mg} / \mathrm{m}^{2}\right.$, iv) administered $3 \mathrm{~h}$ after the MTX infusion. Leucovorin rescue, $15 \mathrm{mg}$ orally or intravenously every $6 \mathrm{~h}$ for 6 times, was started $24 \mathrm{~h}$ after the MTX infusion. Furthermore, leucovorin rescue was continued until the serum MTX concentration was less than $5 \times 10^{-8} \mathrm{~mol} / \mathrm{L}$ if physicians judged there to be a requirement for additional doses. The low-dose 5-FU regimen consisted of a daily infusion of $5-\mathrm{FU}\left(300 \mathrm{mg} / \mathrm{m}^{2} /\right.$ day) [8], while 5 -FU ci consisted of $800 \mathrm{mg} / \mathrm{m}^{2} /$ day for 5 days every 4 weeks. The 5 -FU/l-LV regimen consisted of weekly 5 -FU (500 mg/m ${ }^{2}$, iv) plus $l$-LV $\left(200 \mathrm{mg} / \mathrm{m}^{2}\right.$, iv).

\section{Efficacy}

Among the 92 patients, 86 (93\%) had mild or more severe ascites at initial diagnosis. The remaining $6(7 \%)$ patients had intestinal stenosis without ascites. The response rate in 
Table 1 Patient characteristics

\begin{tabular}{|c|c|c|}
\hline & $N$ & $\%$ \\
\hline \multicolumn{3}{|l|}{ Gender } \\
\hline Male & 53 & 58 \\
\hline Female & 39 & 42 \\
\hline \multicolumn{3}{|l|}{ Age (years) } \\
\hline Median & 59 & \\
\hline Range & $20-77$ & \\
\hline \multicolumn{3}{|l|}{ ECOG performance status } \\
\hline 0 & 2 & \\
\hline 1 & 46 & 50 \\
\hline 2 & 34 & 37 \\
\hline 3 & 10 & 11 \\
\hline \multicolumn{3}{|l|}{ Primary tumor } \\
\hline Present & 69 & 75 \\
\hline Absent & 23 & 25 \\
\hline \multicolumn{3}{|l|}{ Histological type } \\
\hline Diffuse & 77 & 84 \\
\hline Intestinal & 10 & 11 \\
\hline Other, not specified & 5 & 5 \\
\hline \multicolumn{3}{|l|}{ Severe peritoneal metastasis } \\
\hline Massive ascites & 40 & 43 \\
\hline Inadequate oral intake & 34 & 37 \\
\hline Both & 18 & 20 \\
\hline \multicolumn{3}{|l|}{ Ascites $^{\mathrm{a}}$} \\
\hline None & 5 & 5 \\
\hline Mild & 24 & 26 \\
\hline Moderate & 4 & 4 \\
\hline Massive & 58 & 63 \\
\hline \multicolumn{3}{|l|}{ Intravenous drip infusion } \\
\hline Peripheral venous infusion & 24 & 26 \\
\hline Central venous infusion & 28 & 30 \\
\hline
\end{tabular}

ECOG Eastern Cooperative Oncology Group

a One patient did not have pretreatment computed tomography (CT)

patients with ascites was 27\% (95\% CI 17.8-37.4\%): 2 patients achieved disappearance of ascites and 21 patients had a decrease of ascites, 20 patients showed no change of ascites, and 33 patients had an increase of ascites. The remaining 10 patients could not be assessed. Among the 52 patients with inadequate oral intake, improvement was observed in 17 patients (33\%, 95\% CI 20.3-47.1\%), and 2 patients who had undergone endoscopic stent placement or ileostomy during chemotherapy could not be evaluated. The reasons for treatment discontinuation were disease progression in $77(84 \%)$ patients, loss of follow-up in 8 (9\%), death in $2(2 \%)$, unacceptable toxicity in $3(3 \%)$, patient refusal without causal relationship to treatment in 1 (1\%), and physician decision in $1(1 \%)$. Among the 77 patients with disease progression, 57 (74\%) patients had progressive disease associated with peritoneal metastasis.

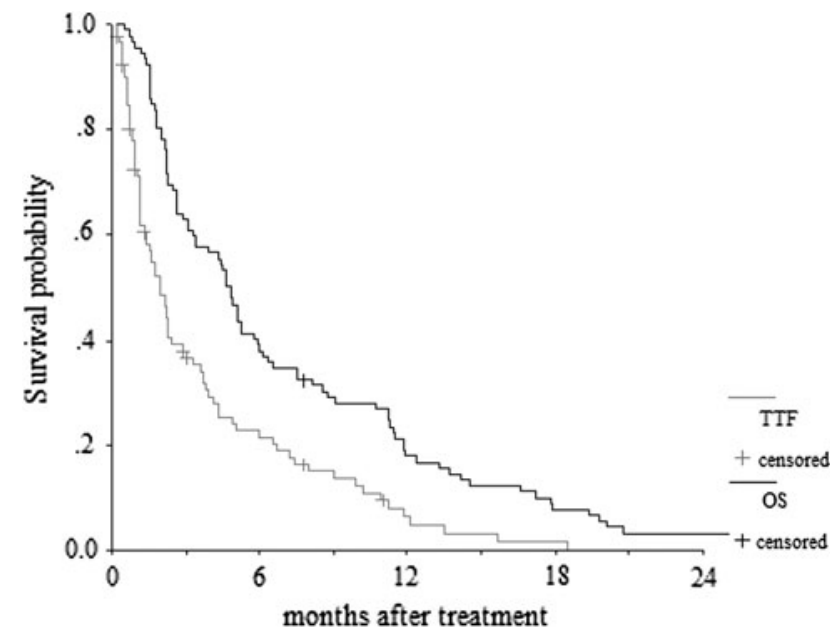

Fig. 1 Overall survival (OS; black line) and time to treatment failure (TTF; gray line) in the 92 patients. The marks on the curves indicate censored cases

At the time of the analysis, 90 patients had died, at a median follow-up time of 4.7 months (range 0.5-45.9 months). Median TTF and OS for the 92 patients in total were 1.9 months (95\% CI 1.3-2.5 months) and 4.6 months (95\% CI 3.9-5.3 months), respectively (Fig. 1).

Second-line treatment

After discontinuation of the first-line treatment, 42 (46\%) patients received supportive care only, $37(40 \%)$ patients received second-line chemotherapy, $1(1 \%)$ patient each received immunotherapy and palliative gastrectomy, and $11(12 \%)$ patients were lost to follow-up. Second-line chemotherapy regimens consisted of taxanes for 28 patients, S-1 (tegafur, 5-chloro-2,4-dihydroxypyridine, and potassium oxonate) for 4 patients, MTX/5-FU for 2 patients, 5-FU ci for 2 patients, and mitomycin for 1 patient. Among the 8 patients who received third-line chemotherapy, 3 received weekly paclitaxel as taxanecontaining regimens. A total of $32(35 \%)$ patients underwent taxane-containing chemotherapy as additional treatment.

\section{Adverse events}

Adverse events in all 92 patients are shown in Table 2. The most common hematological toxicities were neutropenia and anemia. Grade 3 or 4 leukopenia and neutropenia occurred in $17(18 \%)$ and $24(26 \%)$ patients, respectively, and febrile neutropenia occurred in 7 patients. As to nonhematological toxicities, all grades of anorexia were seen in $95 \%$ of patients, while grade 3 nausea and vomiting occurred in 5 and $4 \%$ of patients, respectively. These 
Table 2 Adverse events

\begin{tabular}{|c|c|c|c|c|c|}
\hline & \multicolumn{4}{|c|}{ Number of patients } & \multirow[t]{2}{*}{ Gr 3/4 (\%) } \\
\hline & Gr 1 & Gr 2 & Gr 3 & Gr 4 & \\
\hline \multicolumn{6}{|l|}{ Hematological toxicity } \\
\hline Leukocytes & 26 & 19 & 12 & 5 & 18 \\
\hline Neutrophils & 11 & 18 & 13 & 11 & 26 \\
\hline Hemoglobin & 31 & 33 & 17 & 3 & 22 \\
\hline Platelets & 14 & 2 & 2 & 1 & 3 \\
\hline \multicolumn{6}{|c|}{ Nonhematological toxicity } \\
\hline Anorexia & 33 & 30 & 19 & 5 & 26 \\
\hline Nausea & 50 & 12 & 5 & 0 & 5 \\
\hline Vomiting & 34 & 4 & 4 & 0 & 4 \\
\hline Mucositis & 20 & 2 & 1 & 0 & 1 \\
\hline Diarrhea & 34 & 6 & 3 & 0 & 3 \\
\hline Constipation & 14 & 7 & 2 & 1 & 3 \\
\hline Fatigue & 56 & 27 & 5 & 0 & 5 \\
\hline Febrile neutropenia & - & - & 6 & 1 & 8 \\
\hline Bilirubin & 12 & 4 & 8 & 2 & 11 \\
\hline AST & 27 & 11 & 13 & 0 & 14 \\
\hline ALT & 26 & 12 & 10 & 0 & 11 \\
\hline Creatinine & 12 & 8 & 1 & 0 & 1 \\
\hline
\end{tabular}

Gr grade, $A S T$ aspartate aminotransferase, $A L T$ alanine aminotransferase

gastrointestinal adverse events were frequently attributed to the underlying gastric cancer and peritoneal metastasis. Moreover, elevated levels of bilirubin and transaminase were frequently observed due to progressive disease of peritoneal metastasis. No other unexpected severe toxicities were observed during the treatment. Ten (11\%) patients died within 30 days from the last administration of 5-FU-based chemotherapy, 7 patients died of disease progression, 2 patients died of treatment-related causes, and 1 patient died of intestinal perforation clinically diagnosed as undeniably treatment-related. As to the treatment-related deaths, one patient developed septic shock with febrile neutropenia and another patient had septic shock with no apparent source of infection or neutropenia.

\section{Discussion}

The present study demonstrated that gastric cancer patients with severe peritoneal metastasis receiving 5-FU-based chemotherapy as the first-line treatment had median TTF and OS of 1.9 and 4.6 months, respectively. Early death within 30 days from the last administration of this treatment occurred in $10(11 \%)$ patients, but in the majority of cases, death was caused by disease progression, and treatment-related deaths were observed in only $3(3 \%)$ patients. Therefore, the 5-FU-based chemotherapy for gastric cancer patients with severe peritoneal metastasis was feasible, but its efficacy was unsatisfactory.

Recent Phase III trials have demonstrated the noninferiority of chemotherapy using oral fluoropyrimidines, such as capecitabine or S-1, compared to 5-FU-based chemotherapy $[6,7,9]$. Moreover, oral fluoropyrimidine and cisplatin combination therapy is a standard regimen worldwide [5, 7]. In these clinical trials, gastric cancer patients with severe peritoneal metastasis were excluded from the eligibility criteria, due to the absence of measurable lesions and potential severe complications such as massive ascites, intestinal obstruction, hydronephrosis, and obstructive jaundice.

In a recent Phase III trial (Japan Clinical Oncology Group [JCOG] 0106) comparing 5-FU ci with MTX/5-FU for gastric cancer patients, limited peritoneal metastasis was reported [10]. In that trial, the median survival of 10.6 months in the MTX/5-FU experimental arm did not show a significant advantage over the 9.4 months in the 5-FU ci control arm. Moreover, several Phase II trials of MTX/5-FU, weekly paclitaxel (wPTX), and modified FOLFOX4 for gastric cancer with malignant ascites demonstrated a response rate in patients with ascites of about $35 \%$; median treatment durations of 8,12 , and 10 weeks; and median survivals of 5.1, 5.2, and 8.4 months, respectively [11-13]. Even in these Phase II trials, patients with severe peritoneal metastasis such as massive ascites or inadequate oral intake were almost always excluded from the eligibility criteria. Therefore, we conducted the present retrospective study for patients with severe peritoneal metastasis to determine the indications for chemotherapy and to consider the most suitable regimen.

The prognosis in our patient population was much worse than those in the above-mentioned previous phase II studies for gastric cancer with malignant ascites [11-13], and the efficacy of the 5-FU-based chemotherapy for gastric cancer with severe peritoneal metastasis was not sufficient. This poor result was mainly due to the inferior condition of the patient population (with poor performance status, massive ascites, or inadequate oral intake) compared with the condition of the patients in the previous Phase II studies. Moreover, we consider the low proportion of patients (40\%) receiving second-line chemotherapy as another reason for the poor result in our study. Previous Phase II studies for gastric cancer with malignant ascites did not refer to the post-treatment, while in recent Japanese Phase III trials for advanced gastric cancer, the proportion of patients receiving second-line chemotherapy was $75 \%$ in the SPIRITS (S-1 vs. S-1/cisplatin) trial and $78 \%$ in the JCOG 9912 (5-FU ci vs. S-1 vs. irinotecan/cisplatin) trial $[5,9]$. Although we chose the study patients after May 2001, when paclitaxel was approved for treating gastric cancer in Japan, the present study had a much smaller 
proportion $(40 \%)$ of patients with second-line chemotherapy than that previously observed in any other trials. We think that patients with severe peritoneal metastasis who progressed during the first-line treatment were in extremely poor general condition, so most of the patients might have missed the opportunity to receive second-line chemotherapy.

In the present study none of the patients with severe peritoneal metastasis received an oral fluoropyrimidine- or cisplatin-containing regimen. We believe that these patients could not receive oral fluoropyrimidine plus cisplatin combination therapy for the following reasons: the instability of oral agent administration and absorption, the high risk of complications, and their inability to receive the adequate hydration that is required for renal protection from cisplatin. We need to develop novel chemotherapeutic regimens with non-oral agents, with no need for hydration, and with high feasibility for gastric cancer patients with severe peritoneal metastasis.

Although 5-FU is one of the most commonly used drugs in patients with gastrointestinal malignancies, systemic 5-FU chemotherapy has a limited response rate $[9,10,14]$. Several new cytotoxic agents, such as oxaliplatin and taxanes, have been proven to confer a survival benefit and to show promise as standard anticancer agents for patients with gastric cancer $[6,15,16]$. In Japan, oxaliplatin cannot be used for gastric cancer because it is not yet approved. On the other hand, paclitaxel is recognized as an effective agent for peritoneal metastasis because of its high molecular weight and bulky molecular structure, delaying its clearance from the peritoneal cavity [17, 18]. Currently, there are expectations for the use of paclitaxel as an agent for gastric cancer with peritoneal metastasis, and a randomized Phase II study (JCOG 0407) comparing the best available 5-FU (when the prior chemotherapy included bolus 5-FU, 5-FU ci was administered; for other cases, MTX/5-FU was administered) with wPTX in 5-FUrefractory gastric cancer patients with peritoneal metastasis has completed accrual and awaits final analysis. When paclitaxel was administered alone, it showed a $20-23 \%$ objective response rate in patients with advanced gastric cancer $[19,20]$. In combination chemotherapy, paclitaxel has a synergistic effect in combination with 5-FU and there are no overlapping toxicities. We believe that a regimen with a high response rate against ascites may improve clinical symptoms in the early stage during the course of treatment in gastric cancer patients with severe peritoneal metastasis. Whether high therapeutic efficacy improves the prognosis has not yet been fully defined, and it is necessary to verify which is better: sequential treatment with a 5-FUbased regimen followed by paclitaxel or combined chemotherapy with 5-FU plus paclitaxel. We are conducting a clinical trial of 5-FU/l-LV plus wPTX (FLTAX) for gastric cancer patients with severe peritoneal metastasis, and a feasibility study is currently ongoing as a preliminary step.

In conclusion, 5-FU-based chemotherapy had marginal activity with tolerable toxicity in advanced gastric cancer patients with severe peritoneal metastasis. To achieve a better prognosis, we must investigate new feasible regimens with non-oral agents and no need for hydration for use in this study population.

Acknowledgments We thank Makiko Shinogi for her invaluable assistance in the preparation of this manuscript.

Conflict of interest We have no conflicts of interest to declare.

\section{References}

1. Dupont JB Jr, Lee JR, Burton GR, Cohn I Jr. Adenocarcinoma of the stomach: review of 1497 cases. Cancer. 1987;41:941-7.

2. D'Angelica M, Gonen M, Brennan MF, Turnbull AD, Bains M, Karpeh MS, et al. Patterns of initial recurrence in completely resected gastric adenocarcinoma. Ann Surg. 2004;240:808-16.

3. Yoo CH, Noh SH, Shin DW, Choi SH, Min JS. Recurrence following curative resection for gastric carcinoma. Br J Surg. 2000;87:236-42.

4. Maehara Y, Hasuda S, Koga T, Tokunaga E, Kakeji Y, Sugimachi K. Postoperative outcome and sites of recurrence in patients following curative resection of gastric cancer. Br J Surg. 2000;87:353-7.

5. Koizumi W, Narahara H, Hara T, Takagane A, Akiya T, Takagi $\mathrm{M}$, et al. S-1 plus cisplatin versus S-1 alone for first-line treatment of advanced gastric cancer (SPIRITS trial): a phase III trial. Lancet Oncol. 2008;9:215-21.

6. Cunningham D, Starling N, Rao S, Iveson T, Nicolson M, Coxon $\mathrm{F}$, et al. Capecitabine and oxaliplatin for advanced esophagogastric cancer. N Engl J Med. 2008;358:36-46.

7. Kang YK, Kang WK, Shin DB, Chen J, Xiong J, Wang J, et al. Capecitabine/cisplatin versus 5-fluorouracil/cisplatin as first-line therapy in patients with advanced gastric cancer: a randomized phase III noninferiority trial. Ann Oncol. 2009;20:666-73.

8. Lokich J, Bothe A, Fine N, Perri J. Phase I study of protracted venous infusion of 5-fluorouracil. Cancer. 1981;48:2565-8.

9. Boku N, Yamamoto S, Fukuda H, Shirao K, Doi T, Sawaki A, et al. Fluorouracil versus combination of irinotecan plus cisplatin versus S-1 in metastatic gastric cancer: a randomised phase 3 study. Lancet Oncol. 2009;10:1063-9.

10. Shirao K, Boku N, Yamada Y, Yamaguchi K, Doi T, Takiuchi H, et al. Randomized phase III study of 5-fluorouracil continuous infusion (5FUci) versus methotrexate and 5-FU sequential therapy (MF) in gastric cancer with peritoneal metastasis (JCOG0106). J Clin Oncol. 2009;27:15s (Suppl; Abstr 4545).

11. Yamao T, Shimada Y, Shirao K, Ohtsu A, Ikeda N, Hyodo I, et al. Phase II study of sequential methotrexate and 5-fluorouracil chemotherapy against peritoneally disseminated gastric cancer with malignant ascites: a report from the Gastrointestinal Oncology Study Group of the Japan Clinical Oncology Group, JCOG 9603 Trial. Jpn J Clin Oncol. 2004;34:316-22.

12. Yumiba $\mathrm{T}$, Oba $\mathrm{K}$, Iishi $\mathrm{H}$, Narahara $\mathrm{H}$, Morimoto $\mathrm{T}$, Nakamura $\mathrm{M}$, et al. A multicenter phase II trial of paclitaxel for patients with ascites caused by advanced/recurrent gastric cancer, a new concept of clinical benefit response for non-measurable type of 
gastric cancer. 2008 Gastrointestinal Cancers Symposium (Abstract 51), January 25-27, Orlando, Florida.

13. Oh SY, Kwon HC, Lee S, Lee DM, Yoo HS, Kim SH, et al. A Phase II study of oxaliplatin with low-dose leucovorin and bolus and continuous infusion 5-fluorouracil (modified FOLFOX4) for gastric cancer patients with malignant ascites. Jpn J Clin Oncol. 2007;37:930-5.

14. Ohtsu A, Shimada Y, Shirao K, Boku N, Hyodo I, Saito H, et al. Randomized phase III trial of fluorouracil alone versus fluorouracil plus cisplatin versus uracil and tegafur plus mitomycin in patients with unresectable, advanced gastric cancer: The Japan Clinical Oncology Group Study (JCOG9205). J Clin Oncol. 2003;21:54-9.

15. Al-Batran SE, Hartmann JT, Probst S, Schmalenberg H, Hollerbach S, Hofheinz R, et al. Phase III trial in metastatic gastroesophageal adenocarcinoma with fluorouracil, leucovorin plus either oxaliplatin or cisplatin: a study of the Arbeitsgemeinschaft Internistische Onkologie. J Clin Oncol. 2008;26:1435-42.

16. Van Cutsem E, Moiseyenko VM, Tjulandin S, Majlis A, Constenla M, Boni C, et al. Phase III study of docetaxel and cisplatin plus fluorouracil compared with cisplatin and fluorouracil as firstline therapy for advanced gastric cancer: a report of the V325 Study Group. J Clin Oncol. 2006;24:4991-7.

17. Emi Y, Yamamoto M, Takahashi I, Orita H, Kakeji Y, Kohnoe S, et al. Phase II study of weekly paclitaxel by one-hour infusion for advanced gastric cancer. Surg Today. 2008;38:1013-20.

18. Kobayashi M, Sakamoto J, Namikawa T, Okamoto K, Okabayashi T, Ichikawa $\mathrm{K}$, et al. Pharmacokinetic study of paclitaxel in malignant ascites from advanced gastric cancer patients. World $\mathrm{J}$ Gastroenterol. 2006;12:1412-5.

19. Yamada Y, Shirao K, Ohtsu A, Boku N, Hyodo I, Saitoh H, et al. Phase II trial of paclitaxel by three-hour infusion for advanced gastric cancer with short premedication for prophylaxis against paclitaxel-associated hypersensitivity reactions. Ann Oncol. 2001;12:1133-7.

20. Ohtsu A, Boku N, Tamura F, Muro K, Shimada Y, Saigenji K, et al. An early phase II study of a 3-hour infusion of paclitaxel for advanced gastric cancer. Am J Clin Oncol. 1998;21:416-9. 